\title{
Sensory and perceptual interactions in weight perception
}

\author{
André B. Valdez and Eric L. Amazeen \\ Arizona State University, Tempe, Arizona
}

\begin{abstract}
In four experiments, a multidimensional signal detection analysis was used to determine the influence of length, diameter, and mass on haptically perceived heaviness with and without vision. This analysis allowed us to test for sensory and perceptual interactions between mass and size. As in previous research, sensory interactions were apparent in all four experiments. A novel result was the appearance of perceptual interactions that became more prominent when diameter varied and when vision was allowed. Discussion focuses on how vision and the modalities of touch (i.e., haptic and dynamic) might influence which interactions appear in the data.
\end{abstract}

Perceiving the weight of an object in the hand is common to many real-world and laboratory activities. Despite its ubiquity, though, it is not a simple process. When we grasp an object to judge its weight, we sense its physical properties (e.g., its mass), process this information to form a percept of weight, and then make a decision about how to transform this internal percept into an outward report of heaviness. ${ }^{1}$ These three subprocesses - sensory, perceptual, and decisional - combine to guide our reports of perceived heaviness. These subprocesses also provide three opportunities for features other than mass (e.g., size) to influence perceived heaviness. Disentangling such effects (i.e., determining how each feature influences each subprocess) can be difficult because each can produce equivalent effects on perceptual reports of heaviness. However, contemporary psychophysical techniques, based on signal detection theory, offer a way to distinguish the effects of sensory, perceptual, and decisional subprocesses for multidimensional stimuli. In the present experiments, such multidimensional signal detection techniques were applied in order to determine the influence of length, diameter, and mass on haptically perceived heaviness with and without vision.

\section{Sensory, Perceptual, and \\ Decisional Subprocesses in Weight Perception}

The process of generating a perceptual report of heaviness can be divided into three subprocesses (Amazeen, 1999; Oberle \& Amazeen, 2003). The first is the sensory subprocess, in which the observer detects the physical property or properties relevant to perceiving weight. The second is the perceptual subprocess, in which there is a percept or other psychological or neural code associated with the object's weight. The third is the decisional subprocess, in which the observer uses a criterion or some other rule to generate a perceived heaviness response based on the experienced percept of weight. The fact that multiple processes intervene between stimulus and response presents a major challenge to psychophysical research; namely, a perceptual report is not just a measure of one's percept but represents a combination of, or interaction among, stimulus, percept, and decision. Complicating matters even further is the fact that multiple processes may produce mathematically equivalent distributions of responses (e.g., Cohen, 2003). Psychophysical techniques based on signal detection theory (Green \& Swets, 1966) can be useful in making such distinctions among subprocesses. Figure 1 illustrates these effects in the context of unidimensional signal detection theory. The participant's report of heaviness (in this case, heavier or lighter) is a function of the physical variable mass, the location of the percept along the dimension of perceived weight (measured by $d^{\prime}$ ), and the decision criterion $(C)$ used.

Weight perception presents additional challenges, though, which necessitate the use of methodological and analytical techniques that go beyond unidimensional signal detection theory. The main challenge is that the physical properties relevant to perceived heaviness include more than just the mass of the object; properties such as size (Charpentier, 1891), shape (Dresslar, 1894), and surface material (Ellis \& Lederman, 1999; Flanagan \& Wing, 1997; Flanagan, Wing, Allison, \& Spencely, 1995) have all been shown to have robust influences on perceived heaviness. In the case of size, for example, the well-known size-weight illusion can produce a decrease in perceived heaviness of more than 50\% for stimuli that increase in size without a change in mass (see Stevens \& Rubin, 1970), even when one is aware of the illusion (Flournoy, 1894). Such a strong illusion, combined with the observation that the illusion is influenced by modality (Amazeen, 1997; Ellis \& Lederman, 1993; Oberle \& Amazeen, 2003; Pick \& Pick, 1967), raises the questions of where and how properties such as size interact with mass in the perception of heaviness.

A. B.Valdez, andre.valdez@asu.edu 


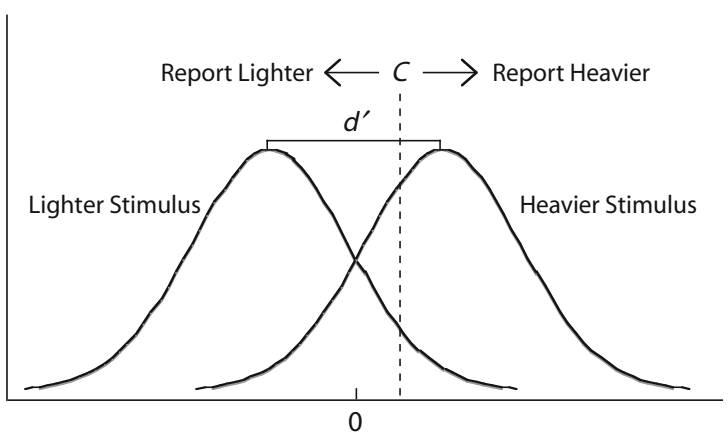

Perception of Weight

Figure 1. Hypothetical perceptual distributions for the perceived weight of two stimuli in a unidimensional signal detection paradigm. Each curve represents the frequency distribution of percepts for each of two stimuli, one light and one heavy. On each trial, the observer compares his or her sampled perception of weight with the decision criterion $(C)$ in order to determine whether to respond lighter or heavier. In signal detection theory, the relative frequencies of correct and incorrect reports can be used to estimate the observer's sensitivity to the difference in weight between the two stimuli $\left(d^{\prime}\right)$ and the location of the decision criterion.

A number of hypotheses have been suggested and explored to explain how size and mass interact. These include an interaction at the level of a physical property such as density (H. E. Ross, 1969; J. Ross \& Di Lollo, 1970; Stevens \& Rubin, 1970) or rotational inertia (Amazeen \& Turvey, 1996), an interaction between internal percepts of size and weight (Anderson, 1970, 1972), or an expectation (Davis \& Roberts, 1976; H. E. Ross \& Gregory, 1970) leading to a reporting or decision bias (Oberle \& Amazeen, 2003). Such hypotheses can be mapped onto the sensory, perceptual, and decisional subprocesses described above. Specifically, each subprocess identifies a place where one feature (e.g., size) may influence the report associated with another (e.g., perceived heaviness). First, a sensory interaction may occur, whereby information about mass is commingled with information about size. This interaction most likely proceeds from an a priori joining of features (see, e.g., the combination of mass and size in the context of density [H. E. Ross, 1969; J. Ross \& Di Lollo, 1970] or rotational inertia [Amazeen, 1997, 1999; Amazeen \& Jarrett, 2003; Amazeen \& Turvey, 1996; Kloos \& Amazeen, 2002]). Second, a perceptual interaction may occur, whereby variations in one percept induce covariations in another percept; such an interaction bears an affinity with percept-percept coupling (Epstein, 1982), as well as with the underlying assumptions of the information integration model of weight perception (Anderson, 1970, 1972). Lastly, a decisional interaction may occur, whereby one feature influences the decision rule that converts the percept associated with the other feature into a report. For example, one may perceive weight on the basis of mass alone yet be led to expect (Davis \& Roberts, 1976; H. E. Ross \& Gregory, 1970) that weight will change as a function of size and, consequently, adopt a decision crite- rion to modify the report of heaviness as a function of size. To identify these interactions, a multidimensional form of signal detection theory is used (see Figure 2).

In multidimensional signal detection theory, stimuli vary along more than one dimension simultaneously. Therefore, each feature (e.g., size) may influence the sensitivity, percept, and decision criteria associated with the other feature (e.g., mass). These influences are represented by the equal probability contours associated with four stimuli (two light, $M_{-}$; two heavy, $M_{+}$; two small, $S_{-}$; and two large, $S_{+}$) in Figure 2. Each equal probability contour identifies the range of percepts (within a given confidence interval) that would occur with repeated presentations of the same stimulus. Note that collapsing these contours onto one axis (e.g., perception of weight alone) produces marginal distributions that are equivalent to the unidimensional case. The shapes, locations, and decision criteria for these contours have been chosen to illustrate a simplified view of each of the three possible sources of interaction described above. First, the elliptical shape of the contours indicates a perceptual interaction in which the perception of weight is correlated with the perception of size. Circular distributions would indicate a lack of a perceptual interaction. Second, the nonrectangular distri-

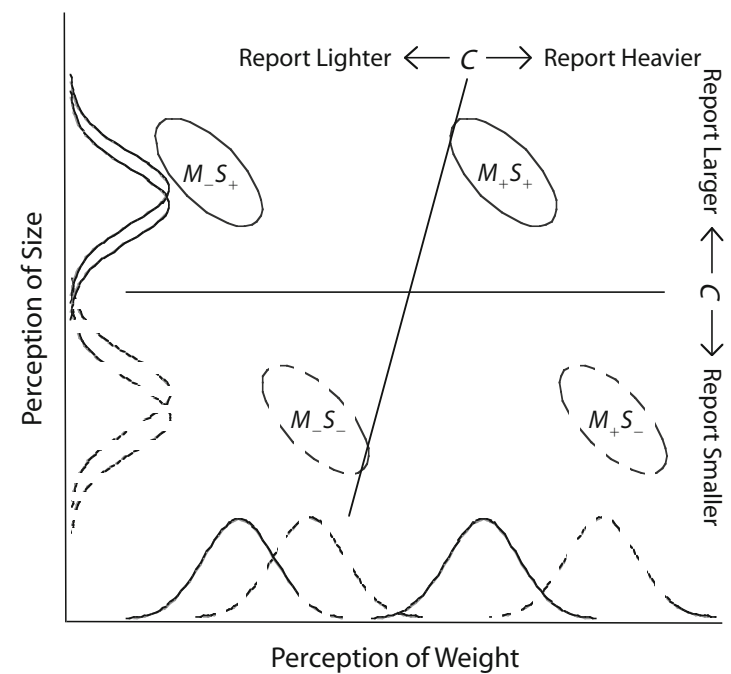

Figure 2. Hypothetical equal probability contours for perceived weight and size in a multidimensional signal detection paradigm. There are four stimuli composed of two levels of mass (light, $M_{-}$, and heavy, $M_{+}$) and two levels of size (small, $S_{-}$, and large, $S_{+}$). Each equal probability contour represents the range of percepts (within a given confidence interval) that would occur with repeated presentations of the same stimulus. On each trial, the observer compares his or her sampled perceptions of size and weight with the decision criterion $(C)$ on each dimension in order to determine whether to respond smaller or larger and lighter or heavier. The shapes and relative locations of the equal probability contours, along with the orientation of the decision criteria, are used to make inferences about sensory, perceptual, and decisional interactions. Each of the three interactions is represented here in a form consistent with the size-weight illusion. 
bution of the four contours (specifically, the fact that the locations of the four contours are laid out as a parallelogram) often indicates a sensory interaction in which an increase in physical size is associated with a decrease in perceived weight. As Cohen (2003) argued, though, such a distribution - technically termed mean shift integralitymay reflect the influence of more than one process. Contours distributed as a square or a rectangle often indicate a lack of sensory interaction. Lastly, the slanted decision criterion for perceived heaviness indicates a decisional interaction in which increased size is associated with a bias toward reporting that the stimulus is lighter. Decision criteria that are orthogonal to the distribution of equal probability contours and to each other would indicate a lack of decisional interaction. Although all three interactions are represented in Figure 2, it is possible (in fact, likely) that only one or two will be present.

Framing the hypotheses regarding the size-weight illusion in the framework of signal detection theory allows us to use multidimensional signal detection analyses to test them (see, e.g., Ashby \& Townsend, 1986; Cohen, 2003; Kadlec, 1995; Kadlec \& Townsend, 1992a, 1992b; Thomas, 1999, 2003; Wickens, 1992). Specifically, the locations and shapes of the equal-probability contours, as well as the decision criteria for perceived size and weight, can be estimated for a set of stimuli that vary in mass and size.

\section{Size, Shape, and Modality}

Such analyses of perceptual independence have been previously applied to weight perception and the size-weight illusion (Amazeen, 1999; Oberle \& Amazeen, 2003). The participants in Oberle and Amazeen grasped small stimuli, whereas the participants in Amazeen (1999) wielded larger objects by a handle (eliminating the opportunity to perceive size by enclosing the stimuli in the hand). In both studies, there were no perceptual interactions present; perceptions of size and weight were independent. When the participants could not see the stimuli, the illusion resulted from a sensory interaction; mass and volume were combined in the tactual information for perceived weight. When the participants could see the stimuli, Oberle and Amazeen found a decisional interaction, suggesting that multiple percepts will be used in making a report of perceived heaviness when they are available and appropriate.

The present experiments expanded these findings by considering the role of shape - specifically, the unique contributions of length and diameter - in weight perception. There was reason to expect that length and diameter would have different effects on perceived heaviness. As early as 1894 , Dresslar showed that changes in shape, without changes in volume, would produce a type of sizeweight illusion that is better termed a shape-weight illusion. This need not suggest, however, that length and diameter each influence perceived heaviness in the same way. In fact, other research has shown that increasing volume by increasing length alone may actually produce an increase in perceived heaviness, a pattern opposite to the classic size-weight illusion (Amazeen, 1997; Ayoub, Mital, Bakken, Asfour, \& Bethea, 1980; Ciriello \& Snook, 1983; Garg \& Badger, 1986; Garg \& Saxena, 1980; Mital \& Fard,
1986). Such differences between length and diameter have been linked to the hypothesis that perceived heaviness is a function of rotational inertia (Amazeen, 1997).

Not only do length and diameter have different effects on perceived heaviness, this difference interacts with modality (Amazeen, 1997). When Amazeen (1997) allowed participants to view the stimuli that they were holding by a handle, increases in both length and diameter produced a decrease in perceived heaviness, yet this effect was stronger for increases in diameter than for increases in length. This suggested that the modalities of vision and touch make unique contributions, wherein touch makes a distinction between changes in length and diameter, whereas vision does not. Similar conclusions have been drawn by Koseleff (1957) and Oberle and Amazeen (2003). The roles of length and diameter were investigated in the present experiments, then, both with and without vision.

\section{Overview}

The present experiments used multidimensional signal detection to determine the influence of length, diameter, and mass on haptically perceived heaviness with and without vision. Participants held stimuli with two levels each of mass and size (either length in Experiments 1 and 2 or diameter in Experiments 3 and 4) and reported their perceived levels on both dimensions. The response frequencies were used to generate multidimensional signal detection models by fitting bivariate Gaussian distributions to the data (Wickens, 1992). These models were used to identify the interactions that may have been present. Although an effect of size should be present in each experiment, it was expected that length and diameter (i.e., shape) would have distinct effects. Comparing the interactions with (Experiments 2 and 4) and without (Experiments 1 and 3) vision allowed us to identify how vision and touch interacted to reshape the flow of perceptual events for the perceiver.

\section{EXPERIMENT 1}

In this experiment, participants held stimuli that varied only in mass and length. The diameters of all the stimuli were equal. Because the participants were not allowed to see the stimuli in their hand (a curtain was used to occlude vision), this experiment assessed the effects of mass and length on haptic weight perception.

\section{Method}

Participants. Fifteen undergraduate students ( 8 of them male, 7 female) at Arizona State University participated in this experiment as a means of fulfilling a course requirement. All the participants were right-handed, and none reported any difficulty with the normal use of their hands or limbs.

Apparatus. The stimulus set was created with a factorial combination of length $(L)$ and mass $(M)$ (uppercase letters are used to denote the physical features, whereas lowercase letters are used to denote the corresponding perceptual report). There were two levels each for mass $\left(M_{-}=24 \mathrm{~g}, M_{+}=30 \mathrm{~g}\right)$ and length $\left(L_{-}=5.8 \mathrm{~cm}\right.$, and $L_{+}=6.0 \mathrm{~cm}$ ) combined to produce the following set of stimuli, $M_{-} L_{-}, M_{-} L_{+}, M_{+} L_{-}$, and $M_{+} L_{+}$. The diameter $(D)$ of each stimulus was $3.0 \mathrm{~cm}$. Mass was altered by drilling lengthwise holes evenly through each cylinder and then distributing lead shot and epoxy evenly from the center to the outer edges. Care was taken to 
eliminate any sound from inside the stimuli and maintain a nearly uniform density. Finally, the ends of each cylinder were capped with balsa wood to conceal any signs of manipulation.

The participants were not permitted to view the stimuli as they grasped them. Accordingly, their right arm extended through a curtain to eliminate all visual cues. This floor-to-ceiling curtain was located to the right of the participants' chair and occluded both the hand and stimulus without causing discomfort or constraining the wielding of the object.

Procedure. Upon entering the experimental room, the participants were seated on a chair beside the curtain. The stimuli were kept out of view. The participants were informed that there were four wooden cylinders (two small, two large, two light, and two heavy) but were otherwise unaware of the nature of the objects. On each of 200 randomly ordered trials (50 trials for each of the four stimuli), the participants were presented a stimulus and were asked to report both perceived length $(l)$ and perceived heaviness $(m)$. Reports were a forced choice on each dimension (i.e., small or large and light or heavy). Thus, there were four possible joint responses per stimulus: $m_{-} l_{-}, m_{-} l_{+}, m_{+} l_{-}$, and $m_{+} l_{+}$. The participants wielded all the stimuli through motions about the wrist. No time limits were imposed, and the participants could choose to pause briefly between trials to avoid fatigue. The procedures in all of the present experiments conformed to the ethical guidelines of the American Psychological Association (2002).

Analysis. The response frequencies for all the participants were pooled to account for the presence of zero frequencies in some cells for some participants (Macmillan \& Creelman, 2005; however, such data should be interpreted with caution due to the increased symmetry in the confusion matrices and the associated possibility that a formal model of these data will not always represent the choice behavior of each individual participant; see Ashby, Maddox, \& Lee, 1994). The main goal of the analysis was to generate a multidimensional signal detection model of the data (as in Figure 2) by fitting bivariate Gaussian distributions to the pooled response frequencies in the manner described by Wickens (1992). These models provided estimates of marginal $d^{\prime}$ (e.g., $d^{\prime}$ for perceived heaviness for only the small stimuli or only the large stimuli) that were equivalent to the estimates generated from the probabilities of hits and false alarms. Statistical comparisons of marginal $d^{\prime}$ were made using a $z$ tes based on estimates of the standard error associated with each signal detection parameter (Gourevitch \& Galanter, 1967; Kadlec, 1995, 1999a, 1999b; Macmillan \& Creelman, 2005; Marascuilo, 1970; Miller, 1996; Robertson \& Morgan, 1990). Statistical evaluations of the correlation between percepts (i.e., the degree of noncircularity in each equal probability contour) were made using the chi-square test of independence. In addition to the multidimensional signal detection analyses, tests of marginal response invariance were conducted to connect the results of the models to traditional observations of the size-weight illusion more fully. The test of marginal response invariance evaluated whether each feature had an effect on the probability of correctly identifying each response. There was a comparison of the probability for each correct report across both levels of the other feature. For example, the probability of correctly reporting that the stimulus felt lighter for the small stimuli was compared with the same probability for the large stimuli. Statistical comparisons of response probabilities were made using a binomial test. In all the tests, the criterion for statistical significance was $p<.05$.

\section{Results and Discussion}

The frequencies of each possible joint response for each of the four stimuli are shown in Table 1. Table 2 shows the results for the test of marginal response invariance. The pairs of probabilities represent the influence of one feature on the probability of correctly reporting each level on the other dimension. Consistent with the size-weight illusion, length had a significant effect on perceived heaviness; as the length increased from the smaller to larger
Table 1

Pooled Response Frequencies for All Four Experiments

\begin{tabular}{lrrrr}
\hline Report & $M_{-} L_{-}$ & $M_{-} L_{+}$ & $M_{+} L_{-}$ & $M_{+} L_{+}$ \\
\hline \multicolumn{5}{c}{ Experiment 1: No Vision } \\
$m_{-} l_{-}$ & 455 & 129 & 148 & 61 \\
$m_{-} l_{+}$ & 80 & 494 & 38 & 172 \\
$m_{+} l_{-}$ & 191 & 31 & 447 & 150 \\
$m_{+} l_{+}$ & 24 & 96 & 117 & 367 \\
\multicolumn{5}{c}{ Experiment 2: Vision Allowed } \\
$m_{-} l_{-}$ & 446 & 95 & 177 & 44 \\
$m_{-} l_{+}$ & 92 & 559 & 38 & 223 \\
$m_{+} l_{-}$ & 196 & 19 & 455 & 82 \\
$m_{+} l_{+}$ & 16 & 77 & 80 & 401 \\
\multicolumn{5}{c}{} \\
$m_{-} d_{-}$ & 451 & 78 & 154 & 16 \\
$m_{-} d_{+}$ & 72 & 556 & 27 & 258 \\
$m_{+} d_{-}$ & 207 & 22 & 515 & 74 \\
$m_{+} d_{+}$ & 17 & 94 & 54 & 402 \\
\multicolumn{5}{c}{ Experiment 4: Vision Allowed } \\
$m_{-} d_{-}$ & 458 & 53 & 109 & 13 \\
$m_{-} d_{+}$ & 86 & 575 & 29 & 233 \\
$m_{+} d_{-}$ & 189 & 22 & 545 & 47 \\
$m_{+} d_{+}$ & 17 & 100 & 57 & 457
\end{tabular}

Note $-M$, mass; $L$, length; $D$, diameter; $m$, perceived heaviness; $l$, perceived length; $d$, perceived diameter. Subscripts for these letters refer to the level of the physical dimension or perceptual report ( - for the lower level, + for the greater level)

stimuli, there was an increased probability of correctly making a lighter response and decreased probability of correctly making a heavier response. Likewise, mass had a significant effect on responses of perceived length; however, the direction of the effect was not consistent. For the smaller stimuli, as mass decreased, there was an increased probability of making a smaller response. For the larger stimuli, as mass decreased, there was an increased probability of making a larger response. Such an effect suggests that the participants were more accurate at lower levels of mass. Table 3 shows the results of the test of marginal $d^{\prime}$. To ascertain how each feature influenced the sensitivity of each response, $d^{\prime}$ was estimated across all levels of each feature. Both paired estimates of $d^{\prime}$ were significantly different. Thus, each feature influenced the sensitivity to the other feature. For perceived length, $d^{\prime}$ was greater for the lighter stimuli, confirming the observation from the marginal response invariance data that the participants were more sensitive to length at lower levels of mass.

These data are more completely represented by fitting bivariate Gaussian distributions to the response frequencies in Table 1 (Wickens, 1992). The resulting model is both presented graphically as equal probability contours in Figure 3 and tabulated as estimates of each parameter and its standard error in Table 4. The model produced the same estimates of marginal $d^{\prime}$ that were generated using the probabilities of hits and false alarms in Table 3. The decision criteria (and their standard errors) in this model were $0.56(0.05)$ for perceived weight and $1.09(0.06)$ for perceived length. The distribution of equal probability contours provides further insight into the source of the observed differences in marginal $d^{\prime}$. As can be seen in Table 4 and Figure 3, as length increased, the distributions 
Table 2

Marginal Response Invariance for All Experiments: Probability of Correctly Reporting Each Level of Mass and Size Across Levels of the Other Dimension

\begin{tabular}{llccccc}
\hline & & \multicolumn{2}{c}{ Length } & & \multicolumn{2}{c}{ Diameter } \\
\cline { 3 - 4 } \cline { 5 - 6 } Report & Stimulus & $\begin{array}{c}\text { No Vision } \\
\text { (Experiment 1) }\end{array}$ & $\begin{array}{c}\text { Vision } \\
\text { (Experiment 2) }\end{array}$ & & $\begin{array}{c}\text { No Vision } \\
\text { (Experiment 3) }\end{array}$ & $\begin{array}{c}\text { Vision } \\
\text { (Experiment 4) }\end{array}$ \\
\hline Lighter & Smaller & .71 & .72 & & .70 & .73 \\
& Larger & .83 & .87 & & .85 & .84 \\
& $z$ & $-5.42^{*}$ & $-7.42^{*}$ & & $-6.70^{*}$ & $-5.25^{*}$ \\
Heavier & Smaller & .75 & .71 & & .76 & .81 \\
& Larger & .69 & .64 & & .64 & .67 \\
& $z$ & $2.71^{*}$ & $2.88^{*}$ & & $5.22^{*}$ & $6.25^{*}$ \\
Smaller & Lighter & .86 & .86 & & .88 & .86 \\
& Heavier & .79 & .84 & & .98 & .88 \\
& $z$ & $3.48^{*}$ & 0.72 & & -0.68 & -1.23 \\
Larger & Lighter & .79 & .85 & & .87 & .90 \\
& Heavier & .72 & .83 & & .88 & .92 \\
& $z$ & $3.10^{*}$ & 0.85 & & -0.78 & -1.35 \\
\hline$* 0.05$ & & & & &
\end{tabular}

along the perceived weight axis shifted by more than two standard errors in the negative direction. In other words, the model revealed that mean perceived weight decreased for the longer stimuli - a pattern consistent with the sizeweight illusion. This pattern suggests some sensory interaction when the participants could hold, but not see, stimuli that varied only in length.

The estimated model parameters in Table 4 reveal another potential source for the size-weight illusion in the present data. Specifically, there was some degree of correlation between perceptions of weight and length for each of the four stimuli. For three out of the four stimuli, the correlation was negative, consistent with the size-weight illusion. To assess whether any of these correlations were statistically significant enough to conclude a perceptual interaction, a chi-square test of independence was used (Thomas, 1995, 2001). ${ }^{2}$ Despite the small degree of correlation revealed by the model, the chi-square test revealed that the responses were statistically independent for each of the four stimuli (all $\chi^{2} \mathrm{~s}<2, p \mathrm{~s}>.05$ ). There was no perceptual interaction in these data.

Conclusions. Experiment 1 revealed how length and mass interact in producing reports of perceived heaviness and size in a purely haptic scenario. A sensory interaction was suggested by the data, but there was no statistically significant evidence of a perceptual interaction; percepts of weight and length were independent. The combined significant effects from the test of marginal response invariance, the test of marginal $d^{\prime}$, and the sheared distribution of equal probability contours all indicated a sensory interaction in which percepts of weight and length were a function of information that included both mass and length. Such an interaction is consistent with the hypothesis that perceived weight and length are a function of the information in rotational inertia, a source of information that combines an object's mass and length. This hypothesis has generally been tested using dynamic touch (see Turvey \& Carello, 1995) but has also been extended to haptic touch (Amazeen \& Jarrett, 2003), the type of touch used in the present experiment.

\section{EXPERIMENT 2}

In this experiment, the participants held the same stimuli as those used in Experiment 1 (varying only in mass and length), but they were allowed to see the stimuli in their hand. This allowed us to determine whether the addition of vision to haptic touch would add to, eliminate, or modify the sensory interaction seen in the purely haptic case in Experiment 1.

\section{Method}

Participants. Fifteen undergraduate students ( 7 of them male, 8 female) at Arizona State University participated in this experiment as a means of fulfilling a course requirement. All the participants

Table 3

Marginal Estimates of $\boldsymbol{d}^{\prime}$ for All Experiments, With $z$ Scores: Estimates for Mass and Size Across Levels of the Other Dimension

\begin{tabular}{clccccc}
\hline \multirow{2}{*}{ Dimension } & & \multicolumn{2}{c}{ Length } & & \multicolumn{2}{c}{ Diameter } \\
\cline { 3 - 4 } \cline { 6 - 6 } Mass & Stimuli & $\begin{array}{c}\text { No Vision } \\
\text { (Experiment 1) }\end{array}$ & $\begin{array}{c}\text { Vision } \\
\text { (Experiment 2) }\end{array}$ & $\begin{array}{c}\text { No Vision } \\
\text { (Experiment 3) }\end{array}$ & $\begin{array}{c}\text { Vision } \\
\text { (Experiment 4) }\end{array}$ \\
& Smaller & 1.24 & 1.14 & & 1.23 & 1.49 \\
& Larger & 1.45 & 1.51 & & 1.36 & 1.43 \\
& $z$ & $-2.06^{*}$ & $-3.61^{*}$ & & -1.33 & 0.59 \\
& Lize & 1.88 & 2.09 & & 2.29 & 2.37 \\
& Heavier & 1.40 & 1.97 & & 2.41 & 2.60 \\
& $z$ & $4.63^{*}$ & 1.11 & & -1.03 & -1.83 \\
\hline${ }^{*} p<05$ & & & & &
\end{tabular}

${ }^{*} p<.05$. 


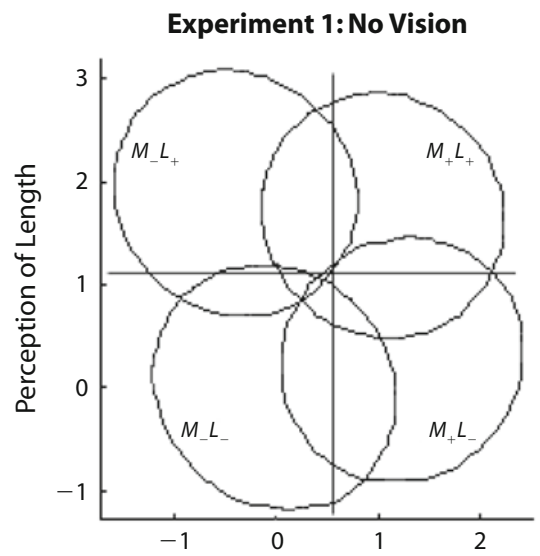

\section{Experiment 2: Vision Allowed}
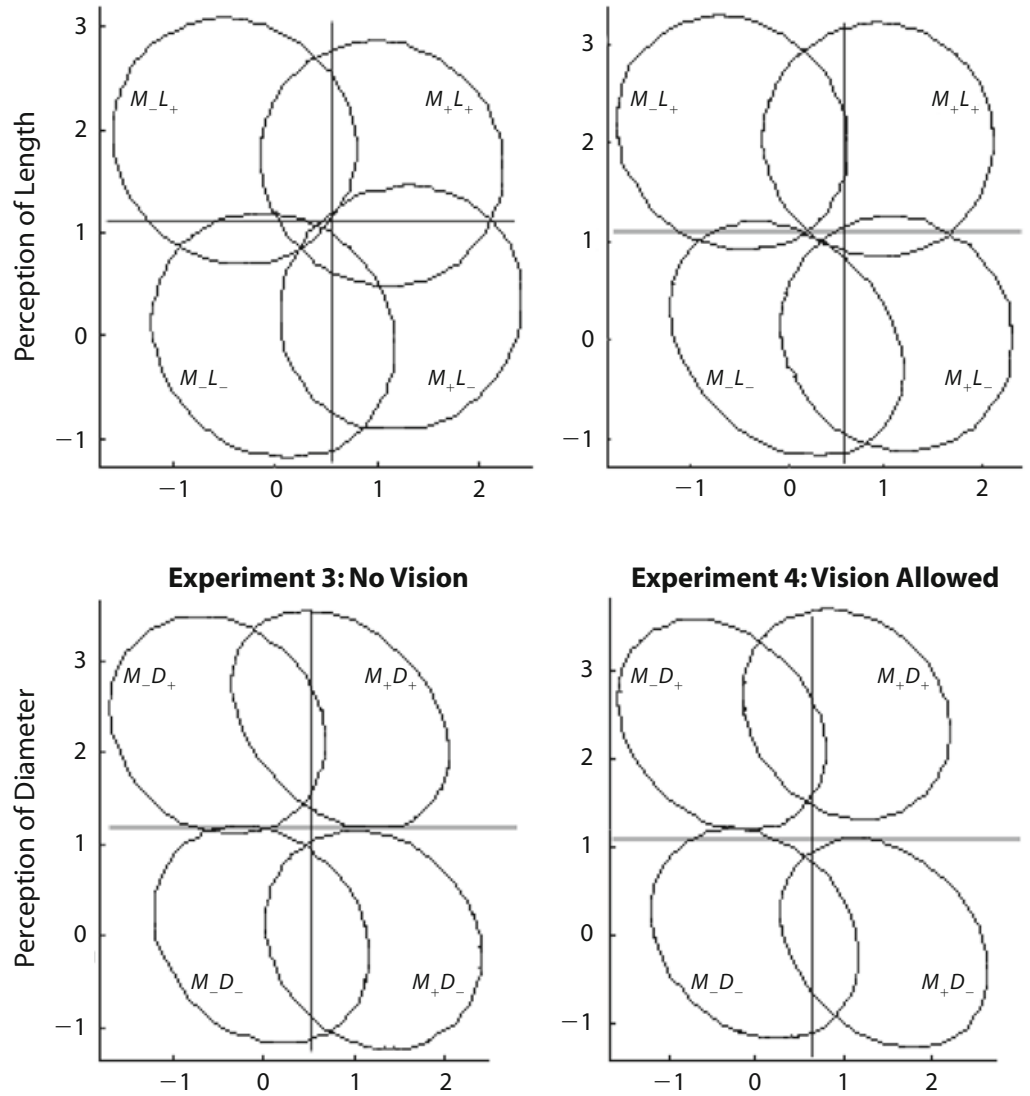

Perception of Weight

Figure 3. Estimated equal probability contours based on the data in Table 1 for all four experiments. In each experiment, there were four stimuli composed of two levels of mass (light, $M_{-}$, and heavy, $M_{+}$) and two levels of either length (short, $L_{-}$, and long, $L_{+}$) or diameter (thin, $D_{-}$, or wide, $D_{+}$). Each equal probability contour represents the range of percepts (within a given confidence interval) that would occur with repeated presentations of the same stimulus. The shapes and relative locations of the equal probability contours are used in the text to make inferences about perceptual and sensory interactions, respectively. These models are also presented in Table 4.

were right-handed, and none reported any difficulty with the normal use of their hands or limbs.

Apparatus. The apparatus in the present experiment was identical to that in Experiment 1, with the following exception. The curtain that was used to occlude the stimuli in the hand was pulled back so that the participants could view the stimuli as they held them in their hand.

Procedure and Analysis. The procedure and analysis in the present experiment were identical to those in Experiment 1, with the following exception. Throughout the trials, the participants were allowed to view the cylinder in their hand. The participants were still not allowed to see the stimuli between trials or to view the entire set of stimuli.

\section{Results and Discussion}

The frequencies of each possible joint response for each of the four stimuli are shown in Table 1. Table 2 shows the results for the test of marginal response invariance. The length of the cylinder had a significant effect on re- ports of perceived heaviness. As the cylinder increased in length, there was an increased probability of correctly making a lighter response and a decreased probability of correctly making a heavier response. Table 3 shows the results of the test of marginal $d^{\prime}$. The paired estimates of $d^{\prime}$ for perceived weight were significantly different, implying a sensory interaction for perceived heaviness. These results are supported by the model presented in Figure 3 and Table 4. The decision criteria (and their standard errors) in this model were $0.57(0.05)$ for perceived weight and $1.06(0.06)$ for perceived length. As in Experiment 1, as length increased, the distributions along the perceived weight axis shifted by more than two standard errors in the negative direction, further indicating a sensory interaction for perceived heaviness. The model also revealed some degree of negative correlation between perceptions of weight and length for all four stimuli. The chi-square 
Table 4

Estimates of the Means and Standard Errors of the Locations and Correlations of the Perceptual Distributions Generated by Fitting Bivariate Gaussian Distributions to the Data in Table 1

\begin{tabular}{|c|c|c|c|c|c|c|}
\hline \multirow[b]{2}{*}{ Stimulus } & \multicolumn{2}{|c|}{$\begin{array}{c}\text { Perceived } \\
\text { Weight }\end{array}$} & \multicolumn{2}{|c|}{$\begin{array}{l}\text { Perceived } \\
\text { Length }\end{array}$} & \multicolumn{2}{|c|}{ Correlation } \\
\hline & $M$ & $S E$ & $M$ & $S E$ & $M$ & $S E$ \\
\hline
\end{tabular}

\begin{tabular}{|c|c|c|c|c|c|c|}
\hline \multicolumn{7}{|c|}{ Experiment 1: No Vision } \\
\hline$M_{-} L_{-}$ & 0.00 & & 0.00 & & -.11 & .0 \\
\hline$M_{-} L_{+}$ & -0.39 & 0.07 & 1.88 & 0.08 & -.07 & .0 \\
\hline$M_{+} L_{-}$ & 1.24 & 0.07 & 0.27 & 0.08 & .06 & .07 \\
\hline$M_{+} L_{+}$ & 1.06 & 0.07 & 1.67 & 0.08 & -.05 & .06 \\
\hline \multicolumn{7}{|c|}{ Experiment 2: Vision Allowed } \\
\hline$M_{-} L_{-}$ & 0.00 & & 0.00 & & -.28 & \\
\hline$M_{-} L_{+}$ & -0.56 & 0.08 & 2.09 & 0.08 & -.11 & .09 \\
\hline$M_{+} L_{-}$ & 1.14 & 0.07 & 0.06 & 0.08 & -.07 & .07 \\
\hline$M_{+} L_{+}$ & 0.94 & 0.07 & 2.02 & 0.08 & -.01 & .07 \\
\hline \multicolumn{7}{|c|}{ Experiment 3: No Vision } \\
\hline$M_{-} D_{-}$ & 0.00 & & 0.00 & & -.20 & .08 \\
\hline$M_{-} D_{+}$ & -0.49 & 0.07 & 2.29 & 0.08 & -.16 & .08 \\
\hline$M_{+} D_{-}$ & 1.23 & 0.07 & -0.06 & 0.09 & -.16 & .08 \\
\hline$M_{+} D_{+}$ & 0.87 & 0.07 & 2.35 & 0.08 & -.32 & .07 \\
\hline \multicolumn{7}{|c|}{ Experiment 4: Vision Allowed } \\
\hline$M_{-} D_{-}$ & 0.00 & & 0.00 & & -.22 & .08 \\
\hline$M_{-} D_{+}$ & -0.38 & 0.07 & 2.37 & 0.08 & -.26 & \\
\hline$M_{+} D_{-}^{+}$ & 1.49 & 0.07 & -0.10 & 0.08 & -.29 & .08 \\
\hline$M_{+} D_{+}$ & 1.04 & 0.07 & 2.50 & 0.09 & -.18 & .09 \\
\hline
\end{tabular}

Note- $M$, mass; $L$, length; $D$, diameter. Subscripts for these letters refer to the level of the physical dimension or perceptual report ( - for the lower level, + for the greater level).

test of independence, though, indicated that the correlation was significant only for one stimulus $\left[M_{-} L_{-}, \chi^{2}(1)=\right.$ $11.26, p<.05$; all others, $\chi^{2}<2, p>.05$ ]. This is weak evidence for a perceptual interaction in these data, possibly only for one stimulus.

Conclusions. Experiment 2 revealed that length had a significant effect on perceived heaviness when the participants could view the stimuli in their hand. As in Experiment 1 , a sensory interaction was suggested. However, there was also some evidence of a perceptual interaction. One possible contributor to the new appearance of a perceptual interaction may have been the increased sensitivity to length accompanying the addition of vision. As can be seen in Table 3, estimates of $d^{\prime}$ increased marginally from 1.88 to $2.09(z=1.92, p<.06)$ and significantly from 1.40 to $1.97(z=5.34, p<.05)$ for the lighter and heavier stimuli, respectively, when vision was added. Improving the sensitivity to length, thereby making it a more salient property to the observer, may have allowed it to have a greater effect on the perceptual processes associated with the size-weight illusion.

\section{EXPERIMENT 3}

In this experiment, the participants held stimuli that varied only in mass and diameter. The lengths of all the stimuli were equal. Because the participants were not allowed to see the stimuli in their hand (a curtain was used to occlude vision), this experiment assessed the effects of mass and diameter on haptic weight perception.

\section{Method}

Participants. Fifteen undergraduate students (6 of them male, 9 female) at Arizona State University participated in this experiment as a means of fulfilling a course requirement. All the participants were right-handed, and none reported any difficulty with the normal use of their hands or limbs.

Apparatus. The apparatus in the present experiment was identical to that in Experiment 1, with the following exception. This stimulus set was created with a factorial combination of mass $(M)$ and diameter $(D)$, whose respective levels were $M_{-}=24 \mathrm{~g}, M_{+}=30 \mathrm{~g}$, $D_{-}=2.8 \mathrm{~cm}$, and $D_{+}=3.0 \mathrm{~cm}$. The length $(L)$ of each stimulus was $6.0 \mathrm{~cm}$.

Procedure and Analysis. The procedure and analysis in the present experiment were identical to those in Experiment 1, with the following exception. This experiment was conducted to obtain reports on the dimensions of diameter and mass, rather than length and mass. As in Experiment 1, a curtain prohibited the participants from viewing the stimulus in their hand.

\section{Results and Discussion}

The frequencies of each possible joint response for each of the four stimuli are shown in Table 1. Table 2 contains the results for the test of marginal response invariance. Consistent with the size-weight illusion, the diameter of the cylinder had a significant effect on reports of perceived heaviness. As the cylinder increased in diameter, there was an increased probability of correctly making a lighter response and decreased probability of correctly making a heavier response. Table 3 contains the results of the test of marginal $d^{\prime}$. Neither of the paired estimates of $d^{\prime}$ was significantly different; there was no influence of either feature on the sensitivity to the other feature. Additional information is contained in the model presented in Figure 3 and Table 4 . The decision criteria (and their standard errors) in this model were $0.52(0.05)$ for perceived weight and 1.18 $(0.06)$ for perceived diameter. As in the previous experiments, as size (in this case, diameter) increased, the distributions along the perceived weight axis shifted by more than two standard errors in the negative direction, suggesting a sensory interaction for perceived heaviness. The model also revealed some degree of negative correlation between perceptions of weight and diameter for all four stimuli. The chi-square test of independence revealed that this correlation was significant for three of the four stimuli $\left[M_{-} D_{-}, \chi^{2}(1)=5.70, p<.05 ; M_{-} D_{+}, \chi^{2}(1)=3.77, p>\right.$ $.05 ; M_{+} D_{-}, \chi^{2}(1)=4.20, p<.05 ; M_{+} D_{+}, \chi^{2}(1)=15.52$, $p<.05]$. These results provide stronger evidence for a perceptual interaction than was seen in the previous two experiments. It was suggested in the previous experiment that the increased evidence for a perceptual interaction in Experiment 2, where vision was allowed, may have followed from the increased sensitivity to size. Similarly, the sensitivity to size (in this case, diameter) was greater in the present experiment than it was in Experiment 1, where length was varied. As can be seen in Table 3, estimates of $d^{\prime}$ increased significantly from 1.88 to 2.29 ( $z=3.62$, $p<.05)$ and from 1.40 to $2.41(z=9.16, p<.05)$ for the lighter and heavier stimuli, respectively.

Conclusions. Experiment 3 showed that diameter had a significant effect on perceived heaviness. As in Experiment 1 , the participants reported that the larger stimuli were lighter than the smaller stimuli. This was at least 
partly due to a sensory interaction of mass and diameter, presumably resulting from the dependence of tactual perception on the information in rotational inertia (Amazeen \& Jarrett, 2003). A notable difference exhibited in the results of the present experiment, though, was the relatively stronger evidence for a perceptual interaction: Perceptions of weight and diameter were significantly correlated for three of the four stimuli, as compared with none in Experiment 1 (where length was varied) and only one of four in Experiment 2 (where length was varied and vision was also allowed). Apparently, the perception of diameter had a stronger effect on the perception of weight than the perception of length did. Insofar as the stronger effects seen in Experiment 2, when vision was allowed, could be related to an increased salience of size as measured through $d^{\prime}$, the still stronger evidence for perceptual interactions in the present experiment may also be related to an increased salience of size (see Table 3). Note that this improved sensitivity to diameter occurred despite its lower range of variation (expressed as a proportion of total diameter), as compared with length. (A more limited range for diameter was chosen precisely because of the increased sensitivity to this dimension shown during pilot testing.) Increasing one's sensitivity to changing size, either through modality or shape, may strengthen a perceptual interaction involved in the size-weight illusion.

\section{EXPERIMENT 4}

In this experiment, the participants held the same stimuli as those used in Experiment 3 (varying only in mass and diameter), but they were allowed to see the stimuli in their hand. This allowed us to determine whether the addition of vision to haptic touch would add to, eliminate, or modify any of the interactions seen in the purely haptic case in Experiment 3.

\section{Method}

Participants. Fifteen students ( 8 of them male, 7 female) at Arizona State University participated in this experiment. All the participants were right-handed, and none reported any difficulty with the normal use of their hands or limbs.

Apparatus. The apparatus in the present experiment was identical to that in Experiment 3, with the following exception. The curtain that was used to occlude the stimuli in the hand was pulled back so that the participants could now view the stimuli as they held them in their hand.

Procedure and Analysis. The procedure and analysis in the present experiment were identical to those in Experiment 3, with the following exception. Throughout the trials, the participants were allowed to view the cylinder in their hand. The participants were still not allowed to see the stimuli between trials or to view the entire set of stimuli.

\section{Results and Discussion}

The frequencies of each possible joint response for each of the four stimuli are shown in Table 1. Table 2 contains the results for the test of marginal response invariance. Consistent with the size-weight illusion, the diameter of the cylinder had a significant effect on reports of perceived heaviness. As the cylinder increased in diameter, there was an increased probability of correctly making a lighter response and a decreased probability of correctly making a heavier response. Table 3 contains the results of the test of marginal $d^{\prime}$. As in Experiment 3, neither of the paired estimates of $d^{\prime}$ was significantly different; there was no influence of either feature on the sensitivity to the other feature. Additional information is contained in the model presented in Figure 3 and Table 4. The decision criteria (and their standard errors) in this model were 0.60 $(0.05)$ for perceived weight and $1.09(0.06)$ for perceived diameter. As in all three previous experiments, as diameter increased, the distributions along the perceived weight axis shifted more than two standard errors in the negative direction, suggesting a sensory interaction for perceived heaviness. The model also revealed some degree of negative correlation between perceptions of weight and diameter for all four stimuli. The chi-square test of independence revealed that this correlation was significant for three of the four stimuli $\left[M_{-} D_{-}, \chi^{2}(1)=7.20, p<.05 ; M_{-} D_{+}\right.$, $\chi^{2}(1)=10.45, p<.05 ; M_{+} D_{-}, \chi^{2}(1)=14.57, p<.05$; $\left.M_{+} D_{+}, \chi^{2}(1)=3.67, p>.05\right]$. As in Experiment 3, these results provide evidence for a perceptual interaction between perceptions of weight and diameter. The increased evidence for a perceptual interaction in this experiment, as compared with Experiment 2, may have followed from the increased sensitivity to size. As can be seen in Table 3, estimates of $d^{\prime}$ increased significantly from 2.09 to 2.37 $(z=2.44, p<.05)$ and from 1.97 to $2.60(z=5.32, p<$ .05 ) for the lighter and heavier stimuli, respectively.

Conclusions. Experiment 4 showed that diameter had a significant effect on reports of heaviness. As has been shown consistently in the present series of experiments, a sensory interaction was present in the data. Also consistent with the other experiments in this series, there was increasing evidence for a perceptual interaction. As in the previous two experiments, this strengthening may be related to the increased salience of size manipulations.

\section{GENERAL DISCUSSION}

The present experiments drew upon multidimensional signal detection to examine the effects of length and diameter on perceived heaviness. Separate experiments were conducted in which the stimuli varied only in length (Experiments 1 and 2) or diameter (Experiments 3 and 4) and in which participants were able to see (Experiments 2 and 4) or not able to see (Experiments 1 and 2) the stimuli in their hand. The goal was to investigate how shape (i.e., length vs. diameter) and modality (i.e., haptic perception with and without vision) influence the interactions that underlie weight perception and the size-weight illusion. The multidimensional signal detection analysis allowed us to test for interactions between mass and size at two levels of processing, sensory and perceptual. The first level concerns interactions in the information; the second, interactions among percepts. A third level, decisionalinteractions that affect the decision rules used to generate each response - was not tested, for the reasons described below; however, there were characteristics in the data to suggest that it should continue to be considered, which will be discussed below. 


\section{Sensory Interactions}

The persistent evidence for a sensory interaction in the size-weight illusion (see also Amazeen, 1999; Oberle \& Amazeen, 2003) suggests that the roles of length and diameter in weight perception are going to be found largely in the information for perceived weight. Research has supported an informational basis to the size-weight illusion based on the information contained in rotational inertia (Amazeen, 1997, 1999; Amazeen \& Jarrett, 2003; Amazeen \& Turvey, 1996; Kloos \& Amazeen, 2002; Shockley, Carello, \& Turvey, 2004; Shockley, Grocki, Carello, \& Turvey, 2001; Turvey, Shockley, \& Carello, 1999). The basic premise of such a model is that size influences weight perception because both mass and volume are combined in determining an object's rotational inertia. A dependence of weight perception on rotational inertia should appear as a sensory interaction between mass and volume.

An inertial model for weight perception and the sizeweight illusion is actually just one instance of a broader model in which the information for many percepts (such as the length, shape, and orientation of a handheld object) is provided in rotational inertia (see the review in Turvey $\&$ Carello, 1995). It is important to note that most of these studies have explored and established the role of rotational inertia by using a type of touch known as dynamic touch (Gibson, 1966). Dynamic touch is the type of touch in which perception is achieved through the use of the muscles exclusively (this is sometimes referred to as the muscle sense; Fitzpatrick, Carello, \& Turvey, 1994). In such studies, then, participants generally hold occluded stimuli, using uniform handles; all of the information for perception is provided by the pattern of resistance (quantified by rotational inertia) that the stimulus presents to the activity of the muscles. In the present experiments, of course, the stimuli did not have handles; the participants grasped the stimuli, enclosing them in their hand. This is a type of touch known as haptic touch (Gibson, 1966). At least one study has shown that participants use rotational inertia to perceive heaviness even when they can wrap their hands around the stimuli (Amazeen \& Jarrett, 2003).

\section{Perceptual Interactions}

A novel feature revealed by the present analyses was the existence of perceptual interactions in the data. Such interactions have long been considered to be part of the size-weight illusion (e.g., Anderson, 1970, 1972), although previous analyses of perceptual independence have failed to show evidence for them (Amazeen, 1999; Oberle \& Amazeen, 2003). However, unlike the sensory interactions, which appeared consistently in each experiment, the perceptual interactions were apparent only in some cases. Specifically, they were more prominent when diameter varied and when the participants could view the stimuli in their hand. The shape of the stimuli and the modality through which they are perceived appear to influence perceptual interaction. However, the key mechanism here may not have actually been shape or modality; as shape and modality were manipulated, the observers' sensitivity to size varied, and conditions of increased sensitivity were associated with stronger perceptual interactions. Anything that results in a greater sensitivity to size may create a better opportunity for the perceiver to combine percepts of size and weight. This suggests that perceptual interactions in the size-weight illusion are persistently possible yet will only transiently appear when the circumstances are favorable.

\section{Decisional Interactions}

Another potential source for the size-weight illusion is a decisional interaction (Oberle \& Amazeen, 2003). Researchers have proposed testing for this type of interaction by comparing paired estimates of marginal $C$ and conditional C (Kadlec, 1995, 1999a, 1999b; Kadlec \& Townsend, 1992a, 1992b). For example, the test of the marginal decision criterion $C$ for perceived heaviness would involve comparing $C$ for the small stimuli with $C$ for the large stimuli. If there is no decisional interaction, one would expect the two criteria to be the same. However, Oberle and Amazeen noticed failures of this test when applied to the size-weight illusion-namely, the decision criterion increased for larger stimuli, suggesting that participants were biased to report that they were lighter. Likewise, when applied to the present data, these tests reveal significant differences in marginal $C$ in the direction predicted by the size-weight illusion for perceived heaviness (but not perceived size) in each of the four experiments.

These data may suggest that the size-weight illusion involves a decisional interaction (at least when the stimuli vary in diameter). However, these tests were not used here because it is not possible to distinguish whether their failure in the present data resulted from a decisional or a perceptual interaction (Ashby \& Townsend, 1986). Because there were not sufficient degrees of freedom to distinguish these two interactions, it was necessary to assume that there was either no decisional interaction or no perceptual interaction in order to generate the models in Figure 3 and Table 4 (Wickens, 1992). It was assumed that there was no decisional interaction for two reasons. First, the participants were told that each stimulus would be presented equally and so were, in effect, instructed to maintain orthogonal decision criteria. Second, theories of the size-weight illusion involving a perceptual interaction are far more common and widely accepted than those involving a decisional interaction, which renders a test of perceptual interaction more interesting and relevant.

Nevertheless, it is important not to discount the real possibility of a decisional interaction here. Although it cannot be tested with the present data, additional experiments using methods that would generate additional degrees of freedom (e.g., confidence ratings) would make such a test possible.

\section{Conclusions}

There are multiple sources for the size-weight illusion. Which source(s) will be present in any given instance, though, is determined by the features of the stimuli and the modality through which they are perceived. In general, an individual will lift an object in order to perceive its weight, thereby employing dynamic touch. Dynamic 
touch, then, will be a constant modality in nearly all lifting situations. When dynamic touch is used, sensory interactions are present to some degree, which is consistent with the notion that perceivers use rotational inertia to perceive weight. However, beyond just lifting the object (say, blindly and with a handle), that same individual may also use haptic touch to enclose the object in the hand(s), vision, or a combination of the two. These additional modalities may improve the perceiver's ability to detect differences in size. Similarly, certain stimuli (say, those that vary in diameter) may possess variations in size that are more easily detected by an observer than are other stimuli (say, those that vary in length). The present results suggest that any characteristic of the stimuli or lifting situation that improves detectability of size will lead to more prominent perceptual interactions. Theories of weight perception, then, should address these two sources for the size-weight illusion in the context of the perceiver's mode of interacting with the stimulus - that is, in the context of the particular perceptual system being used (Gibson, 1966).

\section{AUTHOR NOTE}

This research was supported by National Science Foundation Grant BCS-0518013. The authors thank Robin Thomas for her assistance with the analysis. Correspondence concerning this article should be sent to A. B. Valdez, Department of Psychology, Arizona State University, Box 871104, Tempe, AZ 85287 (e-mail: andre.valdez@asu.edu).

\section{REFERENCES}

AmazeEn, E. L. (1997). The effects of volume on perceived heaviness by dynamic touch: With and without vision. Ecological Psychology, 9, 245-263.

Amazeen, E. L. (1999). Perceptual independence of size and weight by dynamic touch. Journal of Experimental Psychology: Human Perception \& Performance, 25, 102-119.

AMAZEEN, E. L., \& JARRETT, W. D. (2003). The role of rotational inertia in the haptic and haptic + visual size-weight illusions. Ecological Psychology, 15, 317-333.

Amazeen, E. L., \& Turvey, M. T. (1996). Weight perception and the haptic size-weight illusion are functions of the inertia tensor. Journal of Experimental Psychology: Human Perception \& Performance, 22, 213-232.

American Psychological Association (2002). Ethical principles of psychologists and code of conduct. American Psychologist, 57, 1060-1073.

Anderson, N. H. (1970). Averaging model applied to the size-weight illusion. Perception \& Psychophysics, 8, 1-4.

Anderson, N. H. (1972). Cross-task validation of functional measurement. Perception \& Psychophysics, 12, 389-395.

Ashby, F. G., Maddox, W. T., \& LeE, W. W. (1994). On the dangers of averaging across subjects when using multidimensional scaling or the similarity-choice model. Psychological Science, 5, 144-151.

Ashby, F. G., \& Townsend, J. T. (1986). Varieties of perceptual independence. Psychological Review, 93, 154-179.

Ayoub, M. M., Mital, A., Bakken, G. M., Asfour, S. S., \& Bethea, N. J. (1980). Development of strength and capacity norms for manual materials handling activities: The state of the art. Human Factors, 22, 271-283.

Charpentier, A. (1891). Analyse expérimentale de quelques éléments de la sensation de poids [Experimental study of some aspects of weight perception]. Archives de Physiologie Normale et Pathologique, 3, 122-135.

Ciriello, V. M., \& Snook, S. H. (1983). A study of size, distance, height, and frequency effects on manual handling tasks. Human Factors, 5, 473-483.

CoHen, D. J. (2003). Direct estimation of multidimensional perceptual distributions: Assessing hue and form. Perception \& Psychophysics, 65, 1145-1160.

Davis, C. M., \& Roberts, W. (1976). Lifting movements in the sizeweight illusion. Perception \& Psychophysics, 20, 33-36.

Dresslar, F. B. (1894). Studies in the psychology of touch. American Journal of Psychology, 6, 313-368.

Ellis, R. R., \& LeDERMAN, S. J. (1993). The role of haptic versus visual volume cues in the size-weight illusion. Perception \& Psychophysics, 53, 315-324.

Ellis, R. R., \& Lederman, S. J. (1999). The material-weight illusion revisited. Perception \& Psychophysics, 61, 1564-1576.

EPSteIn, W. (1982). Percept-percept couplings. Perception, 11, 75-83.

Fitzpatrick, P., Carello, C., \& Turvey, M. T. (1994). Eigenvalues of the inertia tensor and exteroception by the "muscular sense." Neuroscience, 60, 551-568.

Flanagan, J. R., \& Wing, A. M. (1997). Effects of surface texture and grip force on the discrimination of hand-held loads. Perception \& Psychophysics, 59, 111-118.

Flanagan, J. R., Wing, A. M., Allison, S., \& Spencely, A. (1995). Effects of surface texture on weight perception when lifting objects with a precision grip. Perception \& Psychophysics, 57, 282-290.

FLoURNOY, T. (1894). De l'influence de la perception visuelle des corps sur leur poids apparent [The influence of visual perception on the apparent weight of objects]. L'Année Psychologique, 1, 198-208.

GARG, A., \& BADGER, D. (1986). Maximum acceptable weights and maximum voluntary isometric strengths for asymmetric lifting. Ergonomics, 29, 879-892.

Garg, A., \& SAXENA, U. (1980). Container characteristics and maximum acceptable weight of lift. Human Factors, 22, 487-495.

GiBson, J. J. (1966). The senses considered as perceptual systems. Boston: Houghton Mifflin.

Gourevitch, V., \& Galanter, E. (1967). A significance test for one parameter isosensitivity functions. Psychometrika, 32, 25-33.

Green, D. M., \& Swets, J. A. (1966). Signal detection theory and psychophysics. New York: Wiley.

KADLEC, H. (1995). Multidimensional signal detection analyses (MSDA) for testing separability and independence: A Pascal program. Behavior Research Methods, Instruments, \& Computers, 27, 442-458.

KADLEC, H. (1999a). MSDA 2: Updated version of software for multidimensional signal detection analyses. Behavior Research Methods, Instruments, \& Computers, 31, 384-385.

KADLEC, H. (1999b). Statistical properties of $d^{\prime}$ and $\beta$ estimates of signal detection theory. Psychological Methods, 4, 22-43.

KADlec, H., \& TownSEND, J. T. (1992a). Implications of marginal and conditional detection parameters for the separabilities and independence of perceptual dimensions. Journal of Mathematical Psychology, 36, 325-374.

Kadlec, H., \& Townsend, J. T. (1992b). Signal detection analyses of dimensional interactions. In F. G. Ashby (Ed.), Multidimensional models of perception and cognition (pp. 181-227). Hillsdale, NJ: Erlbaum.

Kloos, H., \& Amazeen, E. L. (2002). Perceiving heaviness by dynamic touch: An investigation of the size-weight illusion in preschoolers. British Journal of Developmental Psychology, 20, 171-183.

KoselefF, P. (1957). Studies in the perception of heaviness: I. Some relevant facts concerning the size-weight-effect (SWE). Acta Psychologica, 13, 242-252.

Macmillan, N. A., \& Creelman, C. D. (2005). Detection theory: A user's guide. Mahwah, NJ: Erlbaum.

Marascuilo, L. A. (1970). Extensions of the significance test for oneparameter signal detection hypotheses. Psychometrika, 35, 237-243.

Miller, J. (1996). The sampling distribution of $d^{\prime}$. Perception \& Psychophysics, 58, 65-72.

Mital, A., \& FARD, H. F. (1986). Psychophysical and physiological responses to lifting symmetrical and asymmetrical loads symmetrically and asymmetrically. Ergonomics, 10, 1263-1272.

Oberle, C. D., \& Amazeen, E. L. (2003). Independence and separability of volume and mass in the size-weight illusion. Perception \& Psychophysics, 65, 831-843.

PICK, H. L., \& PICK, A. D. (1967). A developmental and analytic study of the size-weight illusion. Journal of Experimental Child Psychology, 5, 362-371.

Robertson, C. C., \& Morgan, B. J. T. (1990). Aspects of the design 
and analysis of signal detection experiments. British Journal of Mathematical \& Statistical Psychology, 42, 7-14.

Ross, H. E. (1969). When is a weight not illusory? Quarterly Journal of Experimental Psychology, 21, 346-355.

Ross, H. E., \& GrEGORY, R. L. (1970). Weight illusions and weight discrimination: A revised hypothesis. Quarterly Journal of Experimental Psychology, 22, 318-328.

Ross, J., \& Di Lollo, V. (1970). Differences in heaviness in relation to density and weight. Perception \& Psychophysics, 7, 161-162.

Shockley, K., Carello, C., \& Turvey, M. T. (2004). Metamers in the haptic perception of heaviness and moveableness. Perception \& Psychophysics, 66, 731-742.

Shockley, K., Grocki, M., Carello, C., \& Turvey, M. T. (2001). Somatosensory attunement to the rigid body laws. Experimental Brain Research, 136, 133-137.

Stevens, J. C., \& Rubin, L. L. (1970). Psychophysical scales of apparent heaviness and the size-weight illusion. Perception \& Psychophysics, 8, 225-230.

Thomas, R. D. (1995). Gaussian general recognition theory and perceptual independence. Psychological Review, 102, 192-200.

Tномаs, R. D. (1999). Assessing sensitivity in multidimensional space: Some problems and a definition of a general $d^{\prime}$. Psychonomic Bulletin \& Review, 6, 224-238.

Thомas, R. D. (2001). Perceptual interactions of facial dimensions in speeded classification and identification. Perception \& Psychophysics, 63, 625-650.

Thomas, R. D. (2003). Further considerations of a general $d^{\prime}$ in multidimensional space. Journal of Mathematical Psychology, 47, 220-224.

Turvey, M. T., \& Carello, C. (1995). Dynamic touch. In W. Epstein \& S. Rogers (Eds.), Handbook of perception and cognition: Vol. 5. Perception of space and motion (pp. 401-490). San Diego: Academic Press.
Turvey, M. T., Shockley, K., \& Carello, C. (1999). Affordance, proper function, and the physical basis of perceived heaviness. Cognition, 73, B17-B26.

WiCKENS, T. D. (1992). Maximum-likelihood estimation of a multivariate Gaussian rating model with excluded data. Journal of Mathematical Psychology, 36, 213-234.

\section{NOTES}

1. For the purposes of distinguishing effects in different subprocesses, separate terms will be used for the physical properties, the internal percepts associated with those properties, and the observed reports of the percepts. For weight perception, mass will refer to the physical property, perceived weight will refer to the percept, and reports of perceived heaviness will refer to the report. For size perception, size, length, and diameter will all refer to the physical properties, perceptions of these properties will refer to the percept, and reports of perceived size, length, and diameter will refer to the observed report.

2 . An alternative method for assessing the independence of two reports is the test of sampling independence (Ashby \& Townsend, 1986). This test has been used in previous investigations of the size-weight illusion and has revealed no evidence of a perceptual interaction (Amazeen, 1999; Oberle \& Amazeen, 2003). Likewise, when applied to the data in the present experiments, this test indicates that the reports of heaviness and size were statistically independent for each stimulus in each of the four experiments. Nevertheless, the chi-square test of independence was used here because (1) it provided results more consistent with those of the models in Table 4 and Figure 3 and (2) it possesses greater power to detect smaller correlations.

(Manuscript received October 9, 2006; revision accepted for publication November 26, 2007.) 